\title{
Papier ist geduldig
}

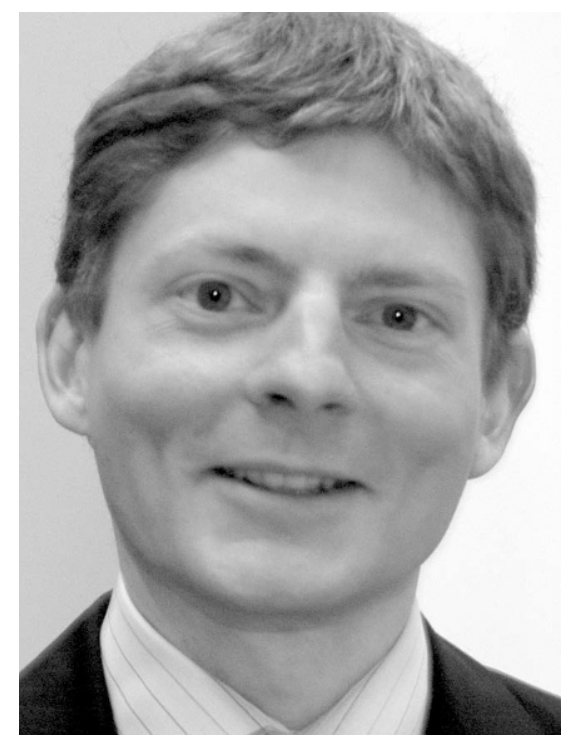

Zertifikate -egal ob freiwilliger Art oder eher erzwungen- erfreuen sich großer „Beliebtheit": Angefangen beim TÜV-Stempel für Kraftfahrzeuge, über Lebensmittelzertifikate bis hin zu Zertifikaten im Bereich der Informationssicherheit spielen sie eine immer bedeutendere Rolle bei der Bewertung der Einhaltung von Normen und Standards. So dienen sie oft gleichzeitig auch als Richtschnur für Verbraucher und Kunden bzw. Auftraggeber, stellen sie doch einen verlässlichen Bewertungsmaßstab dar.

Die Beurteilung, ob einem Zertifikat vertraut werden kann bzw. ob es einen Mehrwert bringt, setzt allerdings voraus, dass die Randbedingungen zur Erteilung desselben bekannt bzw. voll transparent sind und zudem ein fortlaufender, verlässlicher Prozess zur Überprüfung der Einhaltung der Anforderungen besteht. Diese Voraussetzungen sind aber nicht immer erfüllt, oft bleiben beispielsweise die genauen Bewertungsmaßstäbe im Dunkeln.

Auch im Bereich Datenschutz steht mit den Vorgaben in Art. 42 der Datenschutzgrundverordnung (DSGVO) nun die Möglichkeit der Zertifizierung bevor. Daher widmet sich diese Ausgabe der DuD dem Schwerpunkt "Zertifizierung und Standards im Datenschutz". Mit insgesamt sechs Beiträgen beleuchten wir das Thema Zertifizierung im Datenschutz und zeigen gleichzeitig die aktuellen Herausforderungen in diesem Kontext auf.

\section{Die einzelnen Beiträge im Überblick}

Der erste Beitrag "Sinn und Unsinn von Zertifizierungen“ vom Autorenteam um Wilhelm Dolle widmet sich der Frage, ob und unter welchen Randbedingungen Zertifizierungen Sinn ergeben und belastbare Aussagen zulassen.

Der zweite Beitragsblock geht speziell auf eine Zertifizierung nach Art. 42 DSGVO ein und beleuchtet das Thema aus unterschiedlichen Blickwinkeln:

- Henry Krasemann stellt im Beitrag „Der aktuelle Stand der Datenschutz-Zertifizierung und Akkreditierung in Deutschland und Europa" seine persönliche Sicht auf den aktuellen Stand dar und zeigt auf, wie der grundsätzliche Weg zur Akkreditierung aussieht.

- Das Autorenteam von Sönke Maseberg beleuchtet im Beitrag „Der lange Weg zur Akkreditierung nach Art. 42 DSGVO" das Thema aus Sicht eines Antragsstellers. Dabei wird insbesondere auch deutlich, welche Stolperstellen bestehen und wie langwierig das Verfahren (aktuell noch) ist.

- Christian Prietz beschreibt mit seinen Co-Autoren im Beitrag „Prüfung des Datenschutzes"schließlich Komponenten des Prüfverfahrens und differenziert Prüffragen und Prüfanweisungen am Beispiel der "Protokollierung".

- Im Beitrag „Die ISO 27701 und das SDM-V2 im Lichte der Umsetzung der DSGVO“zeigen Martin Rost und Aleksandra Sowa dann auf, welche Hilfestellung diese beiden Standards dem Praktiker geben können.

- Das Autorenteam um Benjamin Heckmann stellt im Beitrag „JOINED-VIV: Umsetzung der DSGVO mittels SDM und unter Einbindung des BSI IT-Grundschutzes" einen verzahnten Ansatz beider Standards vor.

Ergänzt wird der Schwerpunkt dabei durch insgesamt drei weitere Beiträge: „Zertifizierungen im Kontext KRITIScher Infrastrukturen"von Manuel Atug " Der Auskunftsanspruch bei Prüfungsunterlagen" von Judith Klink-Straub und Tobias Straub sowie "Datenschutz im neuen chinesischen Zivilgesetzbuch" von Thomas Hoeren und Stefan Pinelli.

Aufgrund der aktuellen Rechtsprechung des EuGH finden Sie im Bereich „Dokumentation" ergänzend zudem das "FAQ on the judgment of the Court of Justice of the Euro-pean Union in Case C-311/18 - Data Protection Commissioner v Facebook Ireland Ltd and Maximilian Schrems".

Wir haben uns mit den vorliegenden Beiträgen wie immer um eine interessante und repräsentative Auswahl bemüht und hoffen, dass sie Ihnen viele Anregungen für Ihre eigenen Projekte geben. Zusammen mit dem gesamten Herausgeberteam wünsche ich Ihnen als Gastherausgeber eine spannende Lektüre. Und vor allem gilt in diesen außergewöhnlichen Zeiten: Bitte bleiben Sie gesund!

\section{Christoph Wegener}

